Published in final edited form as:

Infect Control Hosp Epidemiol. 2018 July ; 39(7): 884-885. doi:10.1017/ice.2018.91.

\title{
Comparison of Two Glove-Sampling Methods to Discriminate Between Study Arms of a Hand Hygiene and Glove-Use Study
}

\author{
Gwen L. Robinson, MPH ${ }^{1}$, Linda Otieno, MPH ${ }^{1}$, J. Kristie Johnson, PhD $^{1,2}$, Laura J. Rose, \\ MS $^{2}$, Anthony D. Harris, MD, MPH ${ }^{1}$, Judith Noble-Wang, $\mathbf{P h D}^{2}$, and Kerri A Thom, MD, MS ${ }^{1, *}$ \\ for the CDC Epicenter Prevention Program
}

${ }^{1}$ Department of Epidemiology, University of Maryland School of Medicine, Baltimore, MD ${ }^{2}$ Department of Pathology, University of Maryland School of Medicine, Baltimore, Maryland ${ }^{3}$ Division of Healthcare Quality Promotion, National Center for Emerging and Zoonotic Infectious Diseases, Centers for Disease Control and Prevention, Atlanta, GA

\begin{abstract}
In the absence of a gold standard, we compared two glove-sampling methodologies, direct imprint and the sponge stick, to detect a difference between two arms in our study relative to total amount and presence of bacteria.
\end{abstract}

In the absence of a gold standard for sampling gloved hands, this study aims to compare direct imprint versus sponge stick sampling methods to identify an effective glove-sampling method with the ability to detect a difference between the two study arms (Figure).

\section{Methods}

This study, approved by the University of Maryland, Baltimore Institutional Review Board, was performed in two units at the University of Maryland Medical Center in Baltimore, Maryland. This study is imbedded in a randomized trial in which healthcare personnel (HCP) entering Contact Precaution rooms are randomized to either intervention or usual care. Intervention participants are directed by research staff to cleanse gloves with alcoholbased hand rub (ABHR) at each WHO hand hygiene opportunity. ${ }^{1}$ For usual care, HCP behavior at each WHO hand hygiene opportunity is silently recorded. HCP are excluded if they are providing care for patients with Clostridium difficile or if they previously participated. The primary outcomes are (a) total colony forming units (CFU) and (b) presence of pathogenic bacteria.

\footnotetext{
"Corresponding Author: Kerri A. Thom, MD, MS, 685 W Baltimore Street, Bressler Research Building, M021B, Department of Epidemiology and Public Health, University of Maryland School of Medicine, Baltimore, Maryland 21201, Telephone: 410-706-6124; Fax: 410-706-0098, kthom@ som.umaryland.edu.

* Reprint requests: Leslie Norris, 685 W Baltimore Street, MSTF Suite 334E, Department of Epidemiology and Public Health, University of Maryland School of Medicine, Baltimore, Maryland 21201

Conflicts of interest. All authors report no conflicts of interest relative to this article.

CDC Disclaimer: The findings and conclusions in this report are those of the author(s) and do not necessarily represent the official position of the Centers for Disease Control and Prevention/the Agency for Toxic Substances and Disease Registry.
} 
In both study arms, at the last hand hygiene opportunity before exiting the room or after the HCP had completed seven opportunities, gloved hands were sampled to assess bacterial contamination. Each HCP had one hand sampled by the sponge stick method (3M, St. Paul, $\mathrm{MN}$ ) and the other hand by direct imprint of the glove onto a 150mm Tryptic Soy Agar plate (Teknova, Hollister, CA); with the right hand being randomized to receive one or the other method.

In the sponge stick method, the large flat side of the sponge was used to make vertical overlapping "S" strokes and then flipped to make horizontal overlapping "S" strokes along the palmar side of the hands, fingers, and thumb. Next each finger and thumb was sampled by using three upward strokes per digit and then three downward strokes using the opposite thin edge of the sponge. Lastly, using the tip of the sponge, the fingertips were sampled three times each. In the direct imprint method, the research team instructed the HCP to imprint for five seconds their gloved fingertips, thumb, and palm.

Direct agar imprint samples were incubated overnight and colony counts were performed. Sponge stick samples were processed as previously described. ${ }^{2}$ From the eluent, 1/10 dilutions were made. Each dilution was plated on TSA in triplicate for quantitative culturing. Plates were incubated overnight, colonies were counted, and CFU/ml was then calculated.

For each sampling method, CFUs and presence of bacteria were compared across study arms to detect differences between the intervention and usual care arm (Figure). The results from each sampling method were then compared to detect a difference among the differences. For example, we assessed for a difference in total colony counts between intervention and usual care using the sponge stick sampling method and then assessed for a difference using the direct imprint sampling method. Wilcoxon rank-sum tests were used to compare the median distribution of CFUs recovered. The difference between the categories of presence of bacteria was analyzed using a Fisher's Exact Test for each method.

\section{Results}

A total of $42 \mathrm{HCP}$ were enrolled in the study. During each patient encounter, HCP reached a median of three WHO moments before their gloves were sampled. The average time spent sampling for sponge stick was 20s and for agar plate was 13 seconds. When comparing the intervention vs usual care using the direct imprint method, the median CFUs were 2 and 31 respectively $(\mathrm{p}<0.01)$ and for the sponge stick method the median CFU was 1 and 6 respectively $(\mathrm{p}=0.25)$. When comparing the number of gloves positive for bacteria in each of the arms, the direct agar method detected bacteria on 16/25 (64\%) gloves in the intervention and $17 / 17(100 \%)$ gloves in the usual care arm $(\mathrm{p}<0.05)$. Using the sponge stick method, bacteria was detected on 16/25 (64\%) gloves in the intervention and 15/17 (88\%) gloves in the usual care $\operatorname{arm}(\mathrm{p}=0.15)$.

\section{Discussion}

In this study, in the absence of a gold standard, we compared two glove-sampling methodologies, direct imprint and sponge stick, to detect a difference between two arms in our study relative to CFU and presence of bacteria. The direct imprint method could detect a 
significant difference in both outcomes between the intervention and usual care groups, whereas the sponge stick did not.

Prior to this study, there was little data available on microbial sampling of gloved hands and no gold standard exists. The glove-juice method ${ }^{3-5}$ is recognized as a standard for microbial sampling of hands, but not for sampling gloved hands. In the glove-juice method the participant places their hand in a sterile glove and sampling solution is added. The hand is vigorously massaged for 1 minute. The broth cultured for bacteria. The glove-juice method cannot be employed for sampling gloves during patient care as it would not only sample the glove but also bacteria on the HCP hands. The use of this method would also be more disruptive to patient care and likely not tolerated by clinicians during a clinical study.

This study is limited by small sample size; it is possible that with a larger sample a difference between the two study arms would also be detected using the sponge stick method. However, the direct imprint method has a shorter sampling, shorter laboratory processing time, and is a less expensive method, making it preferred. Our data also supports the use of the direct imprint method for the culturing of gloved hands.

\section{Acknowledgments}

Financial support. K.A.T and G.L.R were supported by the Agency for Healthcare Research and Quality (1RO1HS024108-01). K.A.T, A.D.H, G.L.R, and J.K.J were supported by the Center for Disease Control and Prevention (CDC) Prevention Epicenters Program (Grant \# 1U54CK000450-01).

\section{References}

1World Health Organization. [Accessed Nov 20, 2017] Clean Care is Safer Care http://www.who.int/ gpsc/5may/background/5moments/en/

2Rose LJ, Hodges L, O’Connell H, Noble-Wang J. National validation study of a cellulose sponge wipe-processing method for use after sampling Bacillus anthracis spores from surfaces. Appl Environ Microbiol. 2011; 77:8355-8359. [PubMed: 21965403]

3Lingaas E, Fagernes M. Development of a method to measure bacterial transfer from hands. J Hosp Infect. 2009; 72:43-49. [PubMed: 19282052]

4Rosenthal M, Aiello AE, Chenoweth C, et al. Impact of technical sources of variation on the hand microbiome dynamics of healthcare Hs. PLoS One. 2014; 9:e88999. [PubMed: 24551205]

5 Visalachy S, Palraj KK, Kopula SS, Sekar U. Carriage of Multidrug Resistant Bacteria on Frequently Contacted Surfaces and Hands of Health Care Workers. J Clin Diagn Res. 2016; 10:DC18-20. 


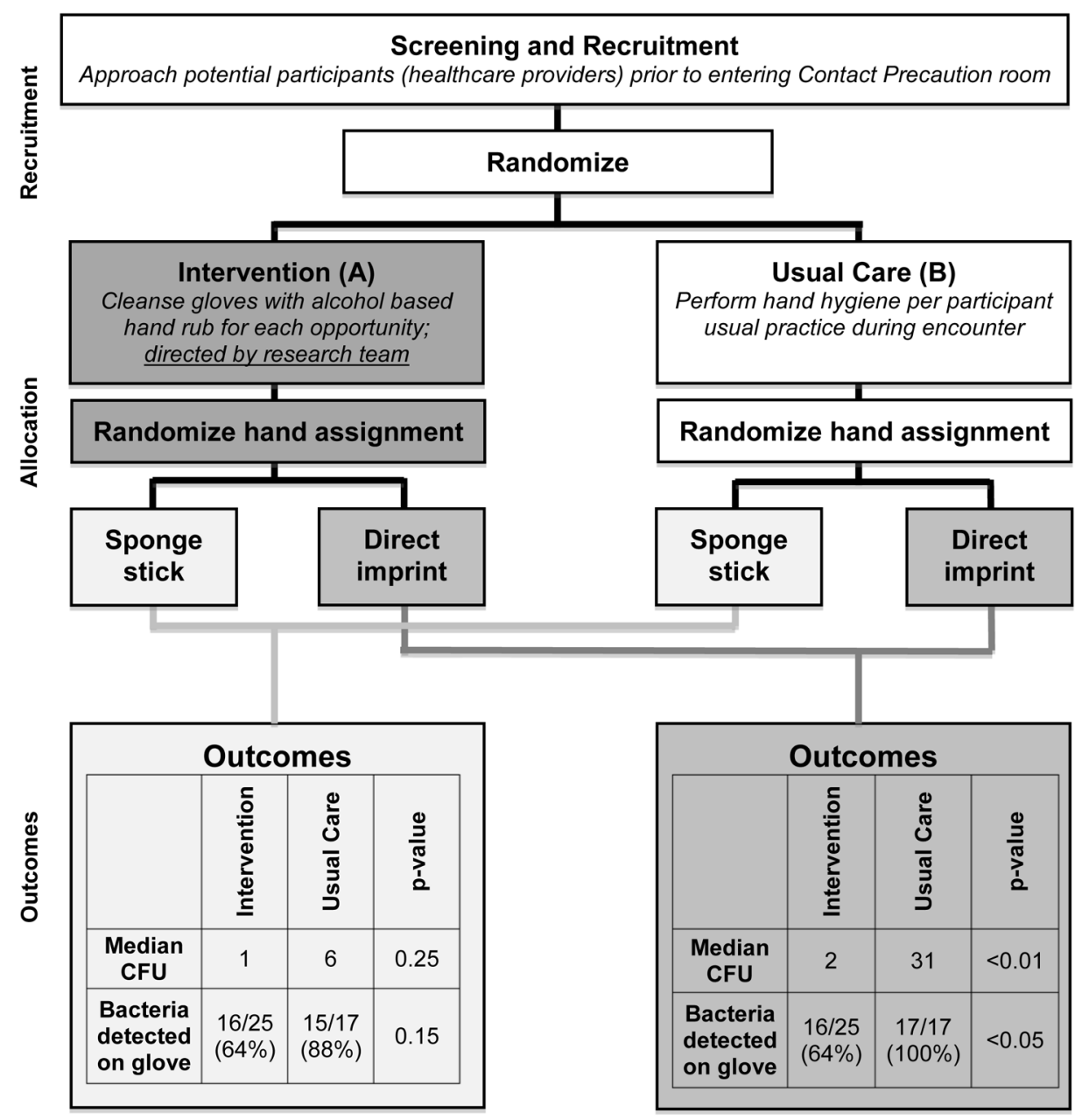

Figure 1.

The sponge stick and the direct imprint method were used to sample each healthcare worker. The outcomes for the intervention and the usual care arm were calculated for the sponge stick and the direct imprint method independently. These outcomes were compared and the method with the ability to detect a difference between the two study arms was chosen as the preferred method for sampling. 